\title{
DEFINITION OF BOUNDARY VALUES OF THICKNESS OF CHROME COVERINGS OF PISTON RINGS
}

\author{
A. D. Sokolov, V. V. Reut, A. A. Shukhat, and A. L. Mil'man
}

UDC 539.3

\begin{abstract}
An approach to the definition of adhesion and cohesion stresses that appear in a piston ring after chroming and its mounting on the piston, its performance in a motor, and corresponding conditions of the critical thicknesses of the covering on the basis of a representation of the ring as a two-layer beam is described.
\end{abstract}

We consider a two-layer beam with a rectangular cross-section of width $b$, the layer of thickness $h_{1}$ is composed of a material with the Young modulus $E_{1}$, and the layer of thickness $h_{2}$ with the Young modulus $E_{2}$. The corresponding sections are denoted by $F_{1}$ and $F_{2}$ (Fig. 1a). The $y$-axis is directed perpendicularly to the lines of division between the layers. It is convenient to take the origin on the $y$-axis in such a way that the relation $E_{1} S_{1}+E_{2} S_{2}=0$ holds, where

$$
S_{\imath}=\int_{F_{i}} y d F, \quad i=1,2
$$

( $S_{i}$ is the statistical moment of the section $F_{i}$ with respect to the central axis $(y=0)$ ). Moreover, the distance between the boundary of the division of the media and the central axis is

$$
a=\left(E_{2} h_{2}^{2}-E_{1} h_{1}^{2}\right) / 2\left(E_{1} h_{1}+E_{2} h_{2}\right)
$$

Standard considerations (see, for instance, [2]) lead to a system of equations that describe a plane bend of the curve of the two-layer beam:

$$
\begin{gathered}
E F \varepsilon_{0}-\left(1+\varepsilon_{0}\right)\left(1 / \rho^{\prime}-1 / \rho\right) E J / \rho=N \\
\left(1+\varepsilon_{0}\right)\left(1 / \rho^{\prime}-1 / \rho\right) E J=M,
\end{gathered}
$$

where $E F=E_{1} F_{1}+E_{2} F_{2}, E J=E_{1} J_{1}+E_{2} J_{2}$, and $M, N$ appear in the cross-sections and are respectively the bending moment and the longitudinal force; $\varepsilon_{0}$ is the magnitude of deformation of the central axis; $\rho$ and $\rho^{\prime}$ are the radii of curvature of the central axis before and after loading, respectively, and finally, $J_{1}$ and $J_{2}$ are the reduced inertia moments of sections with respect to the central axis [2].

Determining the variables $\varepsilon_{0}$ and $\rho^{\prime}$ from (3), we obtain [2] expressions for the normal cohesion stresses on the cross-sections of the beam:

$$
\sigma_{x i}(y)=\left(\dot{E}_{i} / E F\right)(N+M / \rho)+\left(E_{1} / E J\right) M y /(1+y / \rho), \quad i=1,2 .
$$

To find the tangent $\tau_{0}$ and normal $\sigma_{y 0}$ stresses on the boundary of the division between the media (adhesion stresses) we consider an element of the first layer of the beam that corresponds to a segment $d s$ of the central axis (Fig. 1b). It follows from the equilibrium conditions for this element [1] that

$$
\begin{gathered}
\left(d N_{1} / d s\right)-b(\rho+a) / \rho \tau_{0}+\left(Q_{1} / \rho\right)=0 \\
(d Q / d s)-b(\rho+a) / \rho \sigma_{y 0}-\left(N_{2} / \rho\right)-p=0
\end{gathered}
$$

where

$$
N_{1}=\int_{F_{1}} \sigma_{x 1} d F ; \quad Q_{1}=\int_{F_{1}} \tau_{1} d F
$$

Odessa University. Translated from Dinamicheskie Sistemy, No. 9, pp. 72-76, 1990. Original article submitted February 20, 1988. 


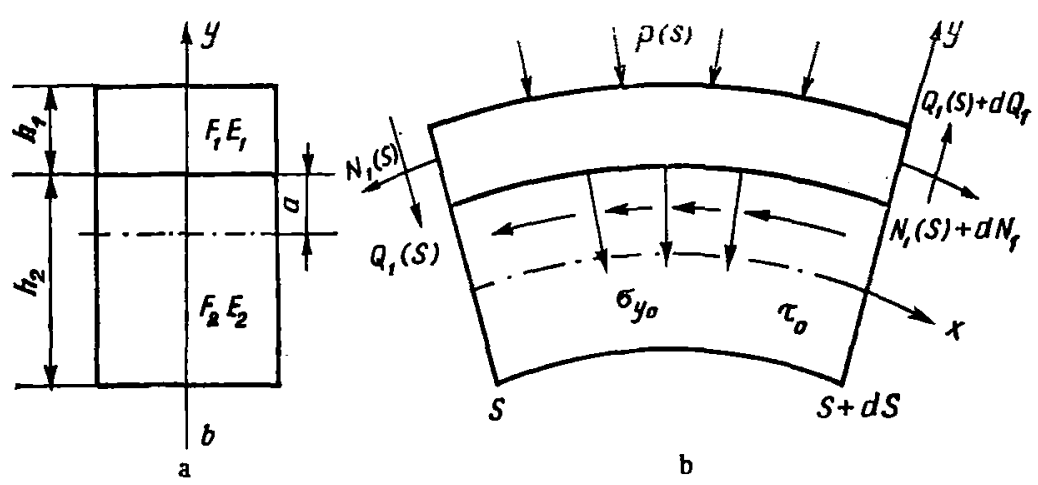

Fig. 1. A rectangular cross-sections of a two-layer beam.

are the longitudinal and transversal forces at the ends of the element; $p(s)$ is an allocated load which is applied to the side surface. The tangent stresses vanish on the surface of the beam. Considering the dependence $\tau_{1}(y)$ as a linear function (it is true for small $h_{1}$, which corresponds to a piston ring with a thin cover), we express $Q_{1}$ by $\tau_{0}$. Then, substituting $N_{1}$ and $Q_{1}$ into (5), where these expressions are found from (6) with regard to (4), we obtain a system of equations to determine $\tau_{0}$ and $\sigma_{y 0}$.

We shall define stresses in the ring after chroming. We assume that the shape of the master form, which is used to produce the piston ring, provides a diagram of radial pressures that appear during the installation of the ring (without covering) in the motor. Considering that the diagram and also the elastic and geometric parameters of the base and of the covering are known, we define stresses that occur in the ring with a covering and which is in a free state. Moreover, we take into account that stresses appear in the covering during the process of making it, and the stresses are related to the form of a crystal net. The appearance of the residual stresses is confirmed by a series of studies, however their form and law of distribution in the covering are not explained completely. Therefore, we count these stresses in the simplest way, namely, we assume that they are constant in the entire covering.

Before making the covering, the stocking is prepared in a holder such that its outer radius coincides with the inner radius $R_{c}$ of the shell of the cylinder. The bending moment which occurs is taken with respect to the central axis of the stocking and is equal to

$$
\hat{M}^{*}(\psi)=\left(R_{c}-h_{2} / 2\right) \int_{0}^{\psi_{\alpha}} p(\xi) \sin (\xi-\psi) d \xi,
$$

where $\psi$ is the angle that is counted from the back of the ring in the present state; $\psi_{\alpha}$ is the angle that corresponds to the end of the ring; $p(\xi)$ is the diagram of radial pressures; and $h_{2}$ is the thickness of the base. Since the residual stresses that appear in the covering are assumed to be uniformly distributed, therefore they are reduced to the following one longitudinal force with respect to the central axis of the covering:

$$
\hat{N}_{i}^{*}=\sigma_{x l}^{*} b h_{1} \text {, }
$$

where $h_{1}$ is the thickness of the covering, $b$ is the height of the ring. Therefore, in the ring with a covering which is embedded into a holder, forces $\hat{M}_{2}$ and $\hat{N}_{1}^{*}$ operate. For the transition of the ring into the free state the opposite stresses should be induced. Since after making the covering the position of the central axis of the ring changes, it is appropriate to reduce the forces $\hat{M}_{2}^{*}$ and $\hat{N}_{1}^{*}$ to corresponding equivalent forces $\left(M_{2}{ }^{*}, N_{2}{ }^{*}\right.$ and $M_{1}{ }^{*}, N_{1}{ }^{*}$, respectively) that are referred to the new central axis.

The unknown parametérs $\psi_{\alpha}$ and $\sigma^{*}{ }_{x 1}$ appear in (7) and (8), and they are defined using the dimensions, which are known, of the lock, stockings, and rings in the free state and expressed in the transcendental equations.

Therefore, it is possible to define stresses $\sigma_{x}$ that occur in the covering as the sum of stresses $\sigma^{*}{ }_{x 1}$ and the stresses from (4), where we set there

$$
M=-\left(M_{1}^{*}+M_{2}^{*}\right), \quad N=-\left(N_{1}^{*}+N_{2}^{*}\right)
$$

The method of finding stresses on the line of adhesive contact has been described earlier. 